\title{
Desenvolvimento Pós-embrionário de Ophyra aenescens (Wiedemann, 1830) (Diptera: Muscidae) em Diferentes Dietas, sob Condições de Laboratório
}

\author{
José Mario d'Almeida/ ${ }^{+}$, Camilla Borges*, Claudia Abrantes Gonçalves*
}
Laboratório de Biologia e Controle de Insetos Vetores, Departamento de Biologia, Instituto Oswaldo Cruz, Av. Brasil 4365, 21045-900 Rio de Janeiro, RJ, Brasil /Laboratório de Entomologia Médica, CCS, UFRJ
*Programa de Vocação Científica, Colégio Politécnico Joaquim Venâncio, Fiocruz

\begin{abstract}
Post-embryonic Development of Ophyra aenescens (Wiedemann, 1830) (Diptera: Muscidae), in Different Diets, under Laboratory Conditions - The performance of various diets (bovine meat, fishsardine, shrimp, dog faeces, and banana) in Ophyra aenescens development was evaluated. The biology was studied in an incubator (BOD) at $27 \pm 1^{\circ} \mathrm{C}$ and $80 \pm 10 \%$ of $\mathrm{RH}$. The developmental time from larvae to adult, the developmental time and viability of larvae and pupae, the weight of pupae as well as the sex ratio of the emerging adults were also determined. Beef and shrimp were the more efficient diets for rearing $\mathrm{O}$. aenescens.
\end{abstract}

Key words: Ophyra aenescens - post-embryonic development - diets

Ophyra aenescens (Wiedemann, 1830) é uma espécie originária das Américas que atualmente tem sido encontrada em várias partes do mundo. $\mathrm{Na}$ década de 1960 foi introduzida na Europa (Hogsette \& Washington 1995). O grau de domiciliação deste muscídeo é bem variado, podendo demonstrar preferência por áreas desabitadas no Rio de Janeiro (d'Almeida 1992) e Cuba (Gregor 1975). Em São Paulo (Linhares 1981) e na América Central (Povolný 1971) a espécie tem sido considerada domiciliada.

Em condições naturais, adultos de $O$. aenescens utilizam diferentes substratos de atração, preferencialmente peixe, embora as larvas se criem com mais frequiência em camarão (d'Almeida 1997).

Sendo os estágios larvais predadores, $O$. aenescens vem assumindo papel de destaque no controle biológico de outras moscas, em especial Musca domestica e Fannia canicularis (Müller 1982, Geden et al. 1988, Farkas e Jantnyik 1990, Turner e Carter 1990).

Apesar da extensa literatura sobre competição e utilização desse muscídeo no controle biológico, são incipientes os trabalhos sobre preferência por dietas larvais (Hogsette e Washington 1995).

A presente pesquisa tem por objetivo comparar e avaliar o desenvolvimento pós-embrionário de $O$. aenescens em laboratório, em diferentes di-

+Autor correspondente. Fax: +55-21-290.1146

Recebido em 1 de abril de 1998

Aceito em 17 de agosto de 1998 etas, visando a possibilidade de utilizá-las no controle biológico de outros dípteros muscóides.

\section{MATERIAIS E MÉTODOS}

As colônias de $O$. aenescens foram estabelecidas a partir de adultos capturados no campus do Instituto Oswaldo Cruz, Rio de Janeiro, RJ, e os trabalhos desenvolvidos no Laboratório de Biologia e Controle de Insetos Vetores do Departamento de Biologia do Instituto.

A manutenção das colônias, obtenção e criação das larvas seguiu a metodologia descrita por d'Almeida e Mello (1995). Os experimentos foram efetuados em câmara climatizada (BOD), regulada para $27 \pm 1^{\circ} \mathrm{C}$ e $80 \pm 10 \%$ UR, no período de janeiro a julho de 1997. As dietas testadas foram carne bovina moída, peixe (sardinha), camarão, fezes caninas frescas e banana fermentada amassada. As carnes foram usadas após $24 \mathrm{~h}$ de exposição ambiente.

Foram utilizadas quatro repetições por dieta ( $1^{\text {a }}$ repetição 80 larvas, $2^{\underline{a}} 100,3^{\underline{a}} 90,4^{\underline{a}} 80$ ), na proporção de duas larvas por grama de dieta, totalizando 350 larvas, provenientes das gerações $\mathrm{F}_{5}$ e $\mathrm{F}_{6}$.

Para avaliar a eficiência das dietas foram usados os parâmetros descritos por d'Almeida e Mello (1995): (a) duração e viabilidade do estádio larvar; (b) duração, viabilidade e peso das pupas (pesadas em grupos de cinco, com $72 \mathrm{~h}$ de formação de pupa); (c) duração e viabilidade do período de larva a adulto; (d) ritmo das emergências e razão sexual; (e) tamanho dos adultos emergidos.

Os resultados foram submetidos à análise de variância (ANOVA), para amostras desiguais e as 
médias comparadas entre si pelo teste de Tukey $(a=0,005)$. A razão sexual foi testada em relação à freqüência esperada $(0,5)$, utilizando-se o teste de aderência do $\mathrm{C}^{2}$, ao nível de $1 \%$ de probabilidade.

\section{RESULTADOS E DISCUSSÃO}

A duração do estádio larvar foi significativamente maior em carne (Tabela I), bem como a maior viabilidade (Tabela II). Tais resultados, ainda que contrastantes, podem indicar que a duração do estádio larvar, em alguns casos, não é um bom indicador da eficiência da dieta.

As larvas podem abandonar precocemente o meio por não encontrarem os nutrientes necessários, ou por excesso de população. Podem, também, permanecer por mais tempo no meio para compensar a baixa quantidade ou qualidade dos nutrientes (Santos e Moya-Borja 1997).

Slansky e Scriber (1985) e Hanski (1987) relataram que as diferenças nas técnicas de acondicionamento do substrato alimentar podem provocar mudanças na textura, $\mathrm{pH}$ e composição bioquímica da dieta, influenciando diretamente o consumo e a utilização de alimento pelas larvas de muscóides. O peixe foi um substrato eficiente para a criação de $O$. aenescens, em condições naturais no Rio de Janeiro (d'Almeida 1997), mas no presente trabalho não foi uma boa dieta, não só pela duração do período larvar, como também pelas baixas viabilidades (Tabelas I e II). Segundo Santos e Moya-Borja (1997), em carnes brancas, as reações bioquímicas desencadeadas pelo processo de decomposição são mais rápidas do que nas carnes vermelhas; o que pode ter influido no desempenho do meio, em condições de laboratório.

A Tabela III indica que não ocorreram diferenças significativas no peso das pupas e no tamanho dos adultos oriundos do camarão e da carne, entretanto, as pupas e os adultos provenientes do pei$\mathrm{xe}$ foram mais leves e menores. Em banana, as lar-

\section{TABELA II}

Viabilidade dos estádios larvar, pupal e do período de larva a adulto, de Ophyra aenescens, em diferentes dietas, sob condições de laboratório, no Rio de Janeiro, RJ

\begin{tabular}{lccc}
\hline & \multicolumn{3}{c}{ Viabilidade $(\%)$} \\
\cline { 2 - 4 } Dieta & Larva & Pupa & Larva/Adulto \\
\hline Carne & 91,14 & 73,35 & 66,57 \\
Camarão & 76,00 & 84,21 & 52,86 \\
Peixe & 62,85 & 4,54 & 2,85 \\
Fezes & 15,71 & 0 & - \\
Banana & 0 & - & - \\
\hline
\end{tabular}

vas não se desenvolveram, embora, segundo Bohart e Gressit (1951) podem ser criadas em matéria orgânica vegetal em decomposição. Hogsette e Washington (1995) só conseguiram o desenvolvimento das larvas de $O$. aenescens em dieta vegetal após a introdução de farinha de carne e osso. Em fezes, a viabilidade larvar foi muito baixa (Tabela II) e as larvas maduras não conseguiram pupar, o que contrasta com as informações de Bohart e Gressit (1951), quando afirmam ser as fezes um dos meios eficientes, entre os vários, de desenvolvimento larvar de 0. aenescens. Outros autores também relataram serem as larvas coprófagas (Nolan e Kissan 1987, Legner e Dietrich 1989, Sulaiman et al. 1990).

Na Figura são apresentados os ritmos de emergência dos adultos. Pode-se observar que os picos ocorreram no $14 \mathrm{o}$ e $15^{\mathrm{o}}$ dias; todavia, os adultos provenientes de larvas criadas em peixe apresentaram emergência mais tardia.

Em carne e camarão a razão sexual foi próxima de 1:1 (Tabela III); no entanto, um aumento do número de fêmeas ocorreu quando o peixe foi utilizado como dieta. Para Fisher (1930), uma razão sexual com desvio não é evolutivamente estável, porque em gerações futuras ocorrerão aumentos

\section{TABELA I}

Duração dos estádios larvar, pupal e do período de larva a adulto de Ophyra aenescens, em diferentes dietas, sob condições de laboratório, no Rio de Janeiro, RJ

\begin{tabular}{|c|c|c|c|}
\hline \multirow{3}{*}{ Dietas } & \multicolumn{3}{|c|}{ Duração (dias) } \\
\hline & Larva & Pupa & Larva/Adulto \\
\hline & $\mathrm{X} \pm \mathrm{DP}$ & $\mathrm{X} \pm \mathrm{DP}$ & $\mathrm{X} \pm \mathrm{DP}$ \\
\hline Carne & $4,97 \pm 0,91(\mathrm{a})^{a}$ & $9,76 \pm 1,11(a)$ & $13,45 \pm 1,10(a)$ \\
\hline Camarão & $4,56 \pm 0,51(b)$ & $9,71 \pm 0,77(a, b)$ & $12,90 \pm 0,94(\mathrm{~b}, \mathrm{~d})$ \\
\hline Peixe & $4,72 \pm 0,86(\mathrm{c}, \mathrm{b})$ & $12,00 \pm 0,97$ (c) & $14,90 \pm 0,84(\mathrm{c})$ \\
\hline Fezes & $4,64 \pm 0,87(\mathrm{~d}, \mathrm{~b})$ & - & - \\
\hline Banana & 0 & - & - \\
\hline
\end{tabular}

X: média; DP: desvio padrão; $a$ : médias com pelo menos uma letra em comum não diferem entre si e as seguidas de letras diferentes diferem significativamente $(\mathrm{a}=0,05)$, pelo teste de comparações múltiplas de Tukey. 


\section{TABELA III}

Peso das pupas, tamanho dos adultos e razão sexual de Ophyra aenescens, em diferentes dietas, sob condições de laboratório, no Rio de Janeiro, RJ

\begin{tabular}{|c|c|c|c|}
\hline & Peso (mg) & Comprimento (mm) & Razão sexual \\
\hline Dietas & $X \pm D P$ & $X \pm D P$ & $c^{2}$ \\
\hline Carne & $13,45 \pm 1,92(a)^{a}$ & $2,00 \pm 0,13(\mathrm{a})$ & $0,504 \mathrm{AA}^{b}$ \\
\hline Camarão & $13,31 \pm 1,74(a)$ & $2,04 \pm 0,11(\mathrm{a})$ & 0,436 AA \\
\hline Peixe & $7,75 \pm 0,71(b)$ & $1,76 \pm 0,08(b)$ & $0,900 \mathrm{AB}$ \\
\hline Fezes & - & - & - \\
\hline Banana & - & - & - \\
\hline
\end{tabular}

X: média; DP: desvio padrão; $a$ : médias com pelo menos uma letra em comum não diferem entre si, e as seguidas de letras diferentes, diferem significativamente $(\mathrm{a}=0,05)$, pelo teste de comparações múltiplas de Tukey; $b$ : letras diferentes indicam desvios significativos da razão sexual esperada $(0,5)$, calculada pelo teste $\mathrm{c}^{2}$, ao nível de $1 \%$ de probabilidade.

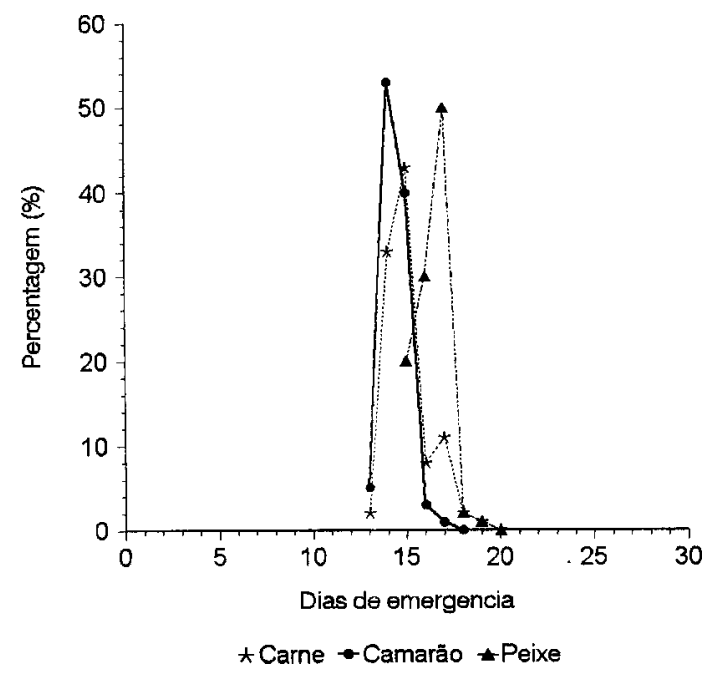

Ritmo de emergência de Ophyra aenescens, criada em diferentes dietas, sob condições de laboratório, no Rio de Janeiro, RJ.

graduais na proporção do sexo observado em menor número. Somente quando a razão sexual for 1:1 haverá estabilidade na população.

$\mathrm{Na}$ Tabela II pode-se constatar que as viabilidades do período de larva a adulto foram baixas, apesar das precauções tomadas para se evitar as interferências da competição larvar, através do fornecimento de quantidades adequadas de alimento. Quanto ao comportamento alimentar das larvas, apesar de serem consideradas como predadoras, elas não são canibais (Skidmore 1985). Segundo esse autor, a umidade do substrato para a formação das pupas é um fator importante para a viabilidade. No presente trabalho, efetuado em câmara climatizada, procurou-se manter a umidade ambiente alta $(80 \pm 10 \%)$.

Utilizando-se como parâmetros as maiores viabilidades, peso das pupas, tamanho dos adultos, além do período mais curto de larva a adulto, podese concluir que a carne bovina moída e o camarão foram as dietas mais eficientes para a criação de O. aenescens em laboratório.

\section{REFERÊNCIAS}

Bohart GE, Gressit JL 1951. Filth-unhabiting flies of Guan. Bull Bernice P Bishop Mus 204: 1-152.

d' Almeida JM 1992. Calyptrate Diptera (Muscidae and Anthomyidae) of the State of Rio de Janeiro - I. Synanthropy. Mem Inst Oswaldo Cruz 87: 381- 386.

d'Almeida JM 1997. Relações tróficas entre dípteros caliptrados (Calliphoridae, Muscidae e Sarcophagidae), criados em diferentes substratos no Rio de Janeiro, RJ. Entomol Vect 4: 111-121.

d'Almeida JM, Mello RP 1995. Eficiência de variadas dietas na criação de Chrysomya megacephala (Fabricius, 1774) e Chrysomya putoria (Wiedemann, 1818) (Diptera: Calliphoridae), sob condições de laboratório. Entomol Vect 2: 95-106.

Farkas R, Jantnyik T 1990. Laboratory studies on Hydrotaea aenescens as a predator of house fly larvae (Diptera: Muscidae). Parasitol Hung 23: 103-108.

Fisher RA 1930. Seleção gênica, p. 276-279. In DJ Futuyama 1993, Biologia Evolutiva, Sociedade Brasileira de Genética, Ribeirão Preto, 631 pp.

Geden CJ, Stinner RE, Axtell RC 1988. Predation by predators of the house fly in poultry manure: effects of predator density, feeding history, interspecific interference, and field conditions. Environ Entomol 17: 320-329.

Gregor F 1975. Synanthropy of Muscidae and Calliphoridae (Diptera) in Cuba. Folia Parasitol (Praha) 22: 57-61.

Hanski T 1987. Nutritional ecology of dung and carrion feeding insects, p. 837-884. In F Slansky Jr, JG Rodrigues (eds), Nutritional Ecology of Insects, Mites, Spider and Related Invertebrates, J Wiley \& Sons, New York.

Hogsette JA, Washington F 1995. Quantitative mass production of Hydrotaea aenescens (Diptera: Muscidae). J Econ Entomol 88: 1238-1242.

Legner EF, Dietrich EJ 1989. Coexistence of predatory Muscina stabulans and Ophyra aenescens (Diptera: 
Muscidae) with dipterous prey in poultry manure. Entomophaga 34: 453-461.

Linhares AX 1981. Synanthropy of Muscidae, Fanniidae and Anthomyidae (Diptera), in the city of Campinas, São Paulo. Rev Bras Entomol 25: 231-243.

Müller P 1982. On the significance of the Musca domestica antagonist Ophyra aenescens (Diptera: Muscidae): III. Laboratory investigation into the interaction between the larvae of Musca domestica and Ophyra aenescens. Angew Parasitol 23: 143154.

Nolan MP, Kissan JB 1987. Nuissance potential of a dump fly, Ophyra aenescens (Diptera: Muscidae), breeding at poultry farms. Environ Entomol 16: 828831.

Povolný D 1971. Synanthropy, p. 17-54. In B Greenberg, Flies and Diseases: Ecology, Classification and Biotic Associations, Vol. 1, Princeton Univ. Press, Princeton.

Santos MJP, Moya-Borja GE 1997. Estudo comparado do desenvolvimento pós-embrionário de Fannia pusio (Wiedemann, 1830) (Diptera: Fanniidae), em diferentes substratos. Arq Biol Tecnol 40: 253-261.

Skidmore P 1985. The Biology of the Muscidae of the World, Junk Publishers, Lancaster, 550 pp.

Slansky Jr F, Scriber H 1985. Food consumption and utilization, p. 87-163. In AA Kerkut, LI Gilbert (eds), Comprehensive Insect Physiology and Pharmacology, Pergamon Press, Oxford.

Sulaiman S, Omar B, Omar S, Jeffrey J, Ghauth L, Busparani V 1990. Survey of microhymenoptera (Hymenoptera: Chalcidoidea) paraziting filth-flies (Diptera Muscidae, Calliphoridae) breeding in refuse and poultry farms in Peninsular Malaysia. J Med Entomol 27: 851-855.

Turner Jr EC, Carter L 1990. Mass rearing and introduction of Ophyra aenescens (Wiedemann, 1830) (Diptera: Muscidae) in light-rise caged layer houses to reduce house-fly populations. J Agric Entomol 7: 247-257. 